\title{
Dipolar Interactions in Superconductor-Ferromagnet
}

\section{Heterostructures}

\author{
Jaime E. Santos ${ }^{1}$, Erwin Frey ${ }^{1,2}$, Franz Schwabl ${ }^{1}$ \\ 1 - Institut für Theoretische Physik T34, Physik-Department der TU München, \\ James-Franck-Straße, 85747 Garching, Germany \\ 2 - Lyman Laboratory of Physics, Harvard University, Cambridge, MA 02138, USA \\ email: jesantos@physik.tu-muenchen.de \\ email: frey@cmts.harvard.edu \\ email: schwabl@physik.tu-muenchen.de
}




\begin{abstract}
We consider a simple model for a superlattice composed of a thin magnetic film placed between two bulk superconductors. The magnetic film is modelled by a planar but otherwise arbitrary distribution of magnetic dipoles and the superconductors are treated in the London approximation. Due to the linearity of the problem, we are able to compute the magnetic energy of the film in the presence of the superconductors. We show that for wavevectors which are much larger than the inverse London penetration depth, the magnetic energy is unchanged with respect to the film in free space, whereas in the case of small wavenumbers compared to the inverse London penetration depth, the magnetic energy resembles the energy of a distribution of magnetisation in a two dimensional space. Possible experimental applications of these results are discussed.
\end{abstract}

75.70.-i, 74.80.-g

Typeset using REVTEX 


\section{INTRODUCTION}

The interplay between superconductivity and ferromagnetism in bulk materials has been the subject of active research since 1957 when Ginzburgt published a paper in which he considered the effect of the field created by a bulk distribution of magnetisation on a superconductor, which was described by the London equations 2 . He has concluded that for a ferromagnetic induction field of the sample larger than its superconducting critical field, this field would destroy superconductivity, but he also pointed out that in thin films or wires where the induction field is much smaller (due to demagnetisation effects) and the critical field higher (due to the small diamagnetic energy) than in bulk superconductors, it should be possible to observe the coexistence of the two phenomena. Experiments carried out by

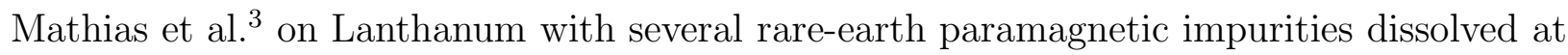
low concentrations suggested that the interaction responsible for the depletion of the superconducting critical temperature of Lanthanum is the exchange interaction between the paramagnetic impurity spins and the superconducting electrons. This interaction induces an effective ferromagnetic interaction between the (antiparallel) spins in the Cooper pair, which tends to destroy it and hence destroy superconductivity. Anderson and Suhl shown that the RKKY interaction between the ferromagnetic spins due to the conduction electrons is significantly reduced in the superconducting state, but pointed out that ferromagnetism could coexist with superconductivity if the ferromagnetic atoms formed small domains. The dependence of the superconducting critical temperature on the concentration of magnetic impurities due to exchange scattering of electrons from these impurities was addressed with the microscopic theory of superconductivity by Abrikosov and Gor'kov日. 
de Gennes and Sarmal have estimated that tipically, the exchange interaction between localised moments and superconducting electrons would be $10^{2}-10^{3}$ larger than the dipolar interaction considered by Ginzburg. The detailed form of the Landau-Ginzburg theory of ferromagnetic superconductors was worked out by Suhl]. Despite their conflicting character, superconductivity and ferromagnetism are seen to coexist in bulk systems, e.g. in $\mathrm{HoMo}_{6} \mathrm{~S}_{8}$ and $\mathrm{ErRh}_{4} \mathrm{~B}_{4}$ 目.

Another system in which the coexistence of superconductivity and ferromagnetism has been observed in the bulk is the nuclear magnet $\mathrm{AuIn}_{2}$ 29. This compound shows a superconducting phase transition at $T_{c}=207 \mathrm{mK}$ and an ordering transition to a ferromagnetic state at an even lower temperature $T_{M}=35 \mu K$. This particularly low temperature can be explained by the weakness of the interaction between the nuclear spins (which is primarily due to an indirect exchange via the conduction electrons).

On a different perspective, the development of epitaxial growth of crystals has permitted the creation of artificial superlattices composed of superconducting and ferromagnetic materials, e.g. $\mathrm{Fe} / \mathrm{V}, \mathrm{Ni} / \mathrm{V}, \mathrm{Ni} / \mathrm{Mo}, \mathrm{EuS} / \mathrm{Pb}, \mathrm{EuO} / \mathrm{Al}$ and $\mathrm{Nb} / \mathrm{Gd}$ [ $\mathrm{E}$. In these superlattices, one can experimentally study the interaction between superconductivity and ferromagnetism when these two effects occur in neighbouring spatial regions and also study the supression of superconductivity as a function of the relative proportion (i.e. layer tickness) of the two materials. More recently, the cuprates $\mathrm{RuSr}_{2} \mathrm{GdCu}_{2} \mathrm{O}_{8-\delta} 202$ and $\mathrm{RuSr}_{2} \mathrm{Gd}_{1+x} \mathrm{Ce}_{1-x} \mathrm{Cu}_{2} \mathrm{O}_{10}$ 24 have been found to show superconductivity and a ferromagnetism below their critical temperatures, $T_{c}=15-40 \mathrm{~K}$ for $\mathrm{RuSr}_{2} \mathrm{GdCu}_{2} \mathrm{O}_{8-\delta}$ and $T_{c}=37 \mathrm{~K}$ (for an optimal $x=0.2$ ) for $\mathrm{RuSr}_{2} \mathrm{Gd}_{1+x} \mathrm{Ce}_{1-x} \mathrm{Cu}_{2} \mathrm{O}_{10}$, the Curie temperatures for magnetic ordering being $T_{M} \approx 133 \mathrm{~K}$ for the first compound and $T_{M} \approx 100 \mathrm{~K}$ for the second. The experimental analysis shows 
that these materials, like all cuprates, have a layered structure and that superconductivity and ferromagnetism seem to occur in different layers. However, a detailed analysis has been hindered by difficulties with the growth of single crystals 25 .

Motivated by such experiments, in which magnetism and superconductivity are seen to occur in different spatial regions of the studied materials, we wish to address the problem of a thin ferromagnetic layer, placed between two bulk superconducting layers (see fig. 11), in which the thickness of the superconducting layers is much larger than the London penetration depth of the superconducting material and the thickness of the ferromagnetic layer is very small compared to this quantity, which is a condition that can be easily obtained with the modern techniques of epitaxial growth 3 . In this limit, the results obtained can also be applied to superlattices of the two materials, given that the ferromagnetic layers are decoupled from one another.

We consider in this paper a simple model system composed of a very thin ferromagnetic film, with an arbitrary distribution of magnetisation in the plane of the film, which is placed in a spatial gap of size $2 d$ between two semi-infinite superconductors described by the London equations. The film is coupled to the superconductors by the electromagnetic interaction, i.e. we neglect the proximity effect 26 and we consider the Josephson current年 flowing between the two superconductors to be zero (the limitations of these approximations will be discussed in section (IV). Having made the approximations indicated above, we are able to solve the problem exactly, by firstly considering the simpler problem of a single dipole in the spatial gap and then superimposing the different solutions, due to the linearity of the London equations. One can then compute the dipolar energy of the distribution of magnetisation. It turns out that for wave-vectors much larger than the inverse London 
penetration depth the form of the dipolar energy in momentum space is unchanged by the presence of the superconductors. On the other hand, for wave vectors much smaller than the inverse London penetration depth, the dipolar energy in momentum space resembles the energy of a distribution of dipoles in a two dimensional space. This behaviour can be traced to the Meissner effect which confines the magnetic flux lines within the spatial gap.

One can think of several possible ways to detect the effects of this change of behaviour of the dipolar interaction at low wave vectors. If one were able to choose the materials composing a layered geometry of superconductor/ferromagnetic film/superconductor in such a way that the Curie transition temperature of the magnetic film to the ferromagnetic state is lower than the critical temperature $T_{c}$ of the superconductor, one should be able to measure the critical properties of the system at the paramagnetic-ferromagnetic transition, in particular such quantities as the specific heat and the magnetic susceptibility, with the superconductors already displaying the Meissner effect and therefore with a modified form of the dipolar energy.

It was shown by Aharony and Fisher 28 that in a $d$-dimensional system, a $d$-dimensional dipolar interaction (such as the one occuring in a bulk tridimensional ferromagnet or in the layered geometry superconductor/ferromagnet/superconductor) is a relevant interaction (in the sense of RG) near a paramagnetic-ferromagnetic transition, leading to a crossover between the critical exponents of the short-range ferromagnet and the critical exponents of the dipolar system when one approaches the critical temperature 2934 . Pelcovits and Halperin 35 have also shown that in the case of a $d$-dimensional systems with a $d+1$-dimensional dipolar interaction (such as the one ocurring in a thin magnetic film film in free space) the universality class of the dipolar system is the same as above. This is due to the fact that, at the 
fixed point, the 'effective' (renormalised) dipolar coupling constant is infinite, making the susceptibility of the system independent of the longitudinal degree of freedom of the magnetisation, which is the one sensitive to the nature of the dipolar interaction. However, in real systems, measurements are not taken exactly at the critical point and one always probes the crossover region. In this region, the dipolar coupling constant is finite and one should be able to detect the distinct character of the transition if the ferromagnetic film is included in a layered geometry with superconductors or if the film is grown in a non-superconducting substrate, due to the different character of the dipolar interaction at small wavevectors. The ideal experiment to detect such a distinction would presumably be a measurement of the longitudinal susceptibility using polarised neutrons 36 . Experiments done with films of EuS/SrS grown on a Si substrate have shown 14 that the low Curie temperature of EuS $(16.5 \mathrm{~K})$ is further reduced in these geometries. The authors of Ref 14 have also performed experiments with films of EuS/Pb, probing the transition between the superconducting state and the normal state in the $\mathrm{Pb}$ layer as a function of the applied magnetic field. Therefore, EuS stands as a good candidate for a material to be used in the ferromagnetic layer. It has the further advantage of being an insulator (see below).

Another possibility would be the study of spin-spin correlation functions in a magnetic film in the ordered phase and outside the critical region. Indeed, Kashuba 11 has shown that the static correlation functions of an XY model with 2d dipolar interactions would display a behaviour analogous to that of the dynamic correlations functions of the stochastic process described the KPZ equation in $1+1$ dimensions 42 , for which the form of these correlation functions is known. An adequate experiment to probe these correlation functions at low momentum compared with the inverse London penetration depth (where such length is 
typically of the order of a thousand Angstroms) would presumably be low-angle neutron scattering from the magnetic fluctuations in a layered geometry. Other possible experiments which could probe the magnetic properties of the system in the ordered phase would be the use of the magnetooptical Kerr effect or of the Faraday effect on samples with a single magnetic layer to image such a layer.

The structure of this paper is as follows: in section $\mathbb{I}$, we define our model in terms of the geometry of the system and the equations which describe it. We also describe the type of boundary conditions we have to consider. In section [II], we present the solution of the equations for a single dipole and construct the solution for a general in-plane distribution

of magnetisation by linear superposition. In section $\mathbb{I V}$, we compute the dipolar energy of the distribution of magnetisation and discuss the physical limitations of the model we have considered. Finally, in section $\mathrm{D}$, we present our conclusions.

\section{GEOMETRY OF THE MODEL AND RELEVANT EQUATIONS}

The geometry of the model is as follows: an infinite distribution of in-plane magnetisation is placed in the plane $z=0$. This distribution is constituted by single magnetic dipoles, placed in an arbitrary fashion with respect to one another (see fig. 2). The in-plane constraint implies that all the dipoles point in a direction within the plane. Above and below the distribution are two bulk superconductors which extend from $z=d$ (respectively $z=-d$ ) to $z=\infty$ (respectively $z=-\infty)$. The spatial gap with size $2 d$ is supposed to be filled with an insulator with magnetic permitivity $\mu_{0}$. The two superconductors are identical and have a magnetic permitivity $\mu$ (i.e. they are paramagnetic, with relative permitivity $\mu_{r}=\mu / \mu_{0}$ ). These supercondutors are described by the London equations (see below), which imply a 
linear relation between the current and the magnetic field.

This linear relation allows us to consider instead a simpler problem, the one of a single magnetic dipole, placed at the origin of the coordinate system and oriented along the $x$ axis. Once this problem has been solved, one can construct the solution for the general case simply by using translational and rotational invariance in the plane and by adding the different solutions. The linearity of the equations will guaranty that the linear combination is also a solution. Furthermore, a uniqueness theorem proved by London $\mathrm{\theta}$ guaranties that this solution is unique.

In the spatial gap, the system is described by the following equations 43 ,

$$
\begin{aligned}
\nabla \times \mathbf{h} & =\mathbf{0} \\
\nabla \cdot \mathbf{b} & =0 \\
\mathbf{b} & =\mu_{0}(\mathbf{h}+\mathbf{m}),
\end{aligned}
$$

where the first two equations are the Maxwell equations for the magnetic field $\mathbf{h}$ and the magnetic induction $\mathbf{b}$ and the third equation is the constitutive relation between the two. For the case of a single dipole oriented along the $x$ axis, the magnetisation $\mathbf{m}(\mathbf{r})=m \hat{\boldsymbol{x}} \delta^{3}(\mathbf{r})$, where $m$ is the magnitude of the magnetic dipole.

The superconductors are described by the equations

$$
\begin{aligned}
\nabla \times \mathbf{h} & =\boldsymbol{\jmath} \\
\mathbf{b} & =-\Lambda \nabla \times \boldsymbol{\jmath}^{s} \\
\mathbf{b} & =\mu \mathbf{h}
\end{aligned}
$$

where the first equation is the Maxwell equation which relates the magnetic field with the 'free' current, the second relation is the second London equation 2 which relates the super- 
current $\boldsymbol{\jmath}^{s}$ with the magnetic induction and the third equation is the constitutive relation between the magnetic induction and the magnetic field. The constant $\Lambda$ is dependent on the type of the superconductor. In a static situation such as the one we are considering, the electric field $\mathbf{e}=\mathbf{0}$ in the superconductor and the total current $\boldsymbol{J}=\boldsymbol{J}^{s}$, i.e. there is no normal component of the current.

Substituting equations (5), (6) in equation (4), one obtains

$$
\begin{aligned}
\nabla^{2} \boldsymbol{\jmath}-\lambda_{L}^{-2} \boldsymbol{\jmath} & =\mathbf{0} \\
\nabla^{2} \mathbf{b}-\lambda_{L}^{-2} \mathbf{b} & =\mathbf{0}
\end{aligned}
$$

where $\lambda_{L}=(\Lambda / \mu)^{1 / 2}$ is the London penetration depth and where equation (8) follows from taking the curl of (7) and using (5) and where we have used the fact that $\nabla \cdot \boldsymbol{\jmath}=0$ (equation of continuity) and $\nabla \cdot \mathbf{b}=0$. These two equations show that the magnetic flux density and the supercurrent penetrate a layer of thickness $\lambda_{L}$ at the surface of the superconductor (Meissner effect).

These equations have to be suplemented by boundary conditions at the surface of the superconductors. These conditions are the continuity of the normal component of $\mathbf{b}$, of the tangential components of $\mathbf{h}$ and of the normal component of the current $\boldsymbol{J}$ at the boundary surfaces of the two superconductors?. If one chooses $\boldsymbol{J}=\nabla \times(g \hat{\boldsymbol{z}})$, where $g(\mathbf{r})$ is a solution of

$$
\nabla^{2} g-\lambda_{L}^{-2} g=0
$$

then one can satisfy the equations (7) and (8) and the boundary condition $\jmath_{z}=0$ at $z= \pm d$. Notice that this choice implies that $y_{z}=0$ throughout the material which is physically reasonable, since $j_{z}=0$ at the surfaces $z= \pm d$ and also for $z= \pm \infty$. 
On the other hand, in the spatial gap, we obtain from equation (1) $\mathbf{h}=-\nabla \Phi_{M}$. Substituting this result in equation (3) and using equation (2), we obtain

$$
\nabla^{2} \Phi_{M}=\nabla \cdot\left(m \hat{\boldsymbol{x}} \delta^{3}(\mathbf{r})\right)
$$

which is Poisson's equation. Since we know the solution of this equation in free space (i.e. in the absence of the superconductors) to obtain the solution in this case, we can write

$$
\Phi_{M}(\mathbf{r})=\frac{m \rho \cos \phi}{4 \pi\left(\rho^{2}+z^{2}\right)^{3 / 2}}+\chi(\rho, \phi, z)
$$

where the first term on the rhs is the solution in free space and the function $\chi(\rho, \phi, z)$ is a solution of the Laplace equation, $\nabla^{2} \chi(\rho, \phi, z)=0$ and where we have used cylindrical polar coordinates for later convenience.

Therefore we need to solve the modified Helmholtz equation (9) in the superconductors and the Laplace equation for $\chi$ in the gap and then fit the two solutions using the continuity conditions for $\mathbf{b}$ and $\mathbf{h}$ at the boundary.

We can further simplify the problem if we notice that the system is invariant under a $\pi$ rotation around the $x$ axis. This invariance imposes the conditions

$$
\begin{array}{r}
g_{-}(\rho, \phi, z)=-g_{+}(\rho,-\phi,-z) \\
\chi(\rho, \phi, z)=\chi(\rho,-\phi,-z)
\end{array}
$$

where $g_{+}$(respectively $g_{-}$) is the solution of the Helmholtz equation in the upper (respectively lower) superconductor. Since the magnetic flux in the superconductor is given in terms of $g$ by 


$$
\begin{aligned}
b_{z} & =\mu \lambda_{L}^{2}\left(\frac{1}{\rho} \frac{\partial}{\partial \rho}\left(\rho \frac{\partial g}{\partial \rho}\right)+\frac{1}{\rho^{2}} \frac{\partial^{2} g}{\partial \phi^{2}}\right) \\
b_{\rho} & =-\mu \lambda_{L}^{2} \frac{\partial^{2} g}{\partial \rho \partial z} \\
b_{\phi} & =-\frac{\mu \lambda_{L}^{2}}{\rho} \frac{\partial^{2} g}{\partial \phi \partial z}
\end{aligned}
$$

and we have, in the spatial gap $\mathbf{b}=\mu_{0}\left(-\nabla \Phi_{M}(\mathbf{r})+m \hat{\boldsymbol{x}} \delta^{3}(\mathbf{r})\right)$, then the continuity conditions for $\mathbf{b}$ and $\mathbf{h}$ imply that at $z=d$,

$$
\begin{aligned}
& \left.\frac{\partial \Phi_{M}}{\partial z}\right|_{z=d}=\mu_{r} \lambda_{L}^{2}\left(\frac{\partial^{2} g_{+}}{\partial^{2} z}-\lambda_{L}^{-2} g_{+}\right)_{z=d} \\
& \left.\frac{\partial \Phi_{M}}{\partial \rho}\right|_{z=d}=\lambda_{L}^{2}\left(\frac{\partial^{2} g_{+}}{\partial \rho \partial z}\right)_{z=d} \\
& \left.\frac{\partial \Phi_{M}}{\partial \phi}\right|_{z=d}=\lambda_{L}^{2}\left(\frac{\partial^{2} g_{+}}{\partial \phi \partial z}\right)_{z=d}
\end{aligned}
$$

where we have used the fact that $g_{+}$is a solution of the Helmholtz equation and that, in the superconductor, $\mathbf{b}=\mu \mathbf{h}$. A similar set of conditions is valid at $z=-d$ but they are trivially related to these conditions by equations (12) and (13).

The above equations and boundary conditions are sufficient to determine the solution of the problem within the London approximation.

\section{THE SINGLE DIPOLE SOLUTION AND THE GENERAL SOLUTION FOR AN ARBITRARY DISTRIBUTION OF MAGNETISATION}

We concluded in the previous section that in order to find the field and current distributions for the case of a single dipole, one needs to find a joint solution of the Laplace and Helmholtz equations, which satisfies the appropriate boundary conditions (17)-(19). Such a solution can be most easily found using cylindrical polar coordinates and is given in terms of Fourier-Bessel integral transforms by 


$$
\begin{aligned}
\Phi_{M}(\rho, \phi, z)= & \frac{m}{4 \pi}\left(\frac{\rho \cos \phi}{\left(\rho^{2}+z^{2}\right)^{3 / 2}}+\int_{0}^{\infty} d k k J_{1}(k \rho) \cosh (k z) \cos \phi\right. \\
& \left.\times \frac{e^{-k d}\left(\mu_{r}^{-1} \sqrt{k^{2}+\lambda_{L}^{-2}}-k\right)}{k \cosh (k d)+\mu_{r}^{-1} \sqrt{k^{2}+\lambda_{L}^{-2}} \sinh (k d)}\right)
\end{aligned}
$$

for $\Phi_{M}(\mathbf{r})$ and

$$
\begin{aligned}
g_{+}(\rho, \phi, z)= & -\frac{m}{4 \pi \mu_{r} \lambda_{L}^{2}} \int_{0}^{\infty} d k k e^{-\sqrt{k^{2}+\lambda_{L}^{-2}}(z-d)} J_{1}(k \rho) \cos \phi \\
& \times \frac{1}{k \cosh (k d)+\mu_{r}^{-1} \sqrt{k^{2}+\lambda_{L}^{-2}} \sinh (k d)}
\end{aligned}
$$

for $g_{+}(\mathbf{r})$ with $g_{-}(\rho, \phi, z)=-g_{+}(\rho,-\phi,-z)$ and where $J_{1}(x)$ is the Bessel function of order one. These integrals can only be calculated explicitly in the particular case $d \rightarrow 0, \mu_{r}=1$. We obtain

$$
\begin{aligned}
\Phi_{M}(\rho, \phi, 0) & =\frac{m}{4 \pi}\left(\frac{1}{\rho \lambda_{L}}+\frac{e^{-\rho / \lambda_{L}}}{\rho^{2}}\right) \cos \phi \\
g_{+}(\rho, \phi, 0) & =-\frac{m \cos \phi}{4 \pi \lambda_{L}^{2} \rho} .
\end{aligned}
$$

The magnetic potential $\Phi_{M}(\rho, \phi, 0)$ corresponds to the magnetic potential of a dipole which produces a $3 \mathrm{~d}$ field at short distances and that at distances $\gg \lambda_{L}$ produces a $2 \mathrm{~d}$ field, i.e. the field produced by a dipole in a two dimensional space. This behaviour can be traced to the Meissner effect, which confines the flux lines to the spatial gap and to a region of size $\lambda_{L}$ in each of the superconductors. Although the $d=0$ case is somewhat unphysical (the superconductor would have to withstand an infinite field), we shall see that as long as $d \ll \lambda_{L}$, this type of behaviour is essentially unchanged.

Now, in order to generalise this solution to the case of an arbitrary distribution of magnetisation, we represent the magnetisation per unit of area in the form

$$
\mathbf{m}(\boldsymbol{\rho})=\sum_{i} \mathbf{m}_{i} \delta^{2}\left(\boldsymbol{\rho}-\boldsymbol{\rho}_{i}\right)
$$


where $\mathbf{m}_{i}$ is a dipole situated at $\boldsymbol{\rho}_{i}=\left(x_{i}, y_{i}\right)$ and $\boldsymbol{\rho}=(x, y)$. In real systems, $\boldsymbol{\rho}_{i}$ will correspond to the sites of a two dimensional lattice where the dipoles are situated. The solution corresponding to this distribution of magnetisation is given by the linear superposition of the solutions corresponding to each $\mathbf{m}_{i}$, i.e.

$$
\begin{aligned}
\Phi_{M}(\rho, \phi, z)= & \sum_{i} \frac{\mathbf{m}_{i} \cdot\left(\boldsymbol{\rho}-\boldsymbol{\rho}_{i}\right)}{4 \pi\left(\left|\boldsymbol{\rho}-\boldsymbol{\rho}_{i}\right|^{2}+z^{2}\right)^{3 / 2}} \\
& +\sum_{i} \frac{\mathbf{m}_{i} \cdot\left(\boldsymbol{\rho}-\boldsymbol{\rho}_{i}\right)}{4 \pi\left|\boldsymbol{\rho}-\boldsymbol{\rho}_{i}\right|} \int_{0}^{\infty} d k k J_{1}\left(k\left|\boldsymbol{\rho}-\boldsymbol{\rho}_{i}\right|\right) \cosh (k z) \\
& \times \frac{e^{-k d}\left(\mu_{r}^{-1} \sqrt{k^{2}+\lambda_{L}^{-2}}-k\right)}{k \cosh (k d)+\mu_{r}^{-1} \sqrt{k^{2}+\lambda_{L}^{-2}} \sinh (k d)}
\end{aligned}
$$

for $\Phi_{M}(\mathbf{r})$ and

$$
\begin{aligned}
g_{+}(\rho, \phi, z)= & -\sum_{i} \frac{\mathbf{m}_{i} \cdot\left(\boldsymbol{\rho}-\boldsymbol{\rho}_{i}\right)}{4 \pi \mu_{r} \lambda_{L}^{2}\left|\boldsymbol{\rho}-\boldsymbol{\rho}_{i}\right|} \int_{0}^{\infty} d k k J_{1}\left(k\left|\boldsymbol{\rho}-\boldsymbol{\rho}_{i}\right|\right) e^{-\sqrt{k^{2}+\lambda_{L}^{-2}}(z-d)} \\
& \times \frac{1}{k \cosh (k d)+\mu_{r}^{-1} \sqrt{k^{2}+\lambda_{L}^{-2}} \sinh (k d)}
\end{aligned}
$$

for $g_{+}(\mathbf{r})$. The function $g_{-}(\mathbf{r})$ is constructed from the single dipole solution in an analogous manner. Note that one cannot use the equations (12) and (13) because we no longer have the $\pi$ rotation symmetry around the $x$ axis 44 . Using the magnetic potential $\Phi_{M}(\mathbf{r})$, we can now compute the dipolar energy of the system.

\section{THE MAGNETIC ENERGY OF THE SYSTEM}

The dipolar energy of the system can be obtained by substituting the dipole distribution by an equivalent distribution of loops of current, i.e. one which will produce the same field distribution. The easiest way to compute the energy necessary for the formation of such a current distribution is to compute it with the currents of the individual loops which form the 
distribution kept constant. The dipolar energy of the magnetisation distribution is equal to the energy necessary to form the current distribution when the fluxes in each loop are kept constant, which is minus the energy computed with constant currents and is given by 4,4

$$
E_{m}=-\frac{1}{2} \mu_{0} \int d^{2} \rho \mathbf{m}(\boldsymbol{\rho}) \cdot \mathbf{h}(\rho, \phi, z=0)
$$

Substituting $\mathbf{h}=-\nabla \Phi_{M}$, with $\Phi_{M}(\mathbf{r})$ given by (25) and approximating the discrete sums on the lattice by integrals, we obtain

$$
\begin{aligned}
E= & \frac{\mu_{0}}{8 \pi} \int_{B Z} \frac{d^{2} k}{(2 \pi)^{2}} \frac{2 \pi}{k}(\mathbf{k} \cdot \mathbf{m}(\mathbf{k}))(\mathbf{k} \cdot \mathbf{m}(-\mathbf{k})) \times \\
& \frac{\left(\mu_{r}^{-1} \sqrt{k^{2}+\lambda_{L}^{-2}}+k\right) e^{k d}+\left(\mu_{r}^{-1} \sqrt{k^{2}+\lambda_{L}^{-2}}-k\right) e^{-k d}}{\left(\mu_{r}^{-1} \sqrt{k^{2}+\lambda_{L}^{-2}}+k\right) e^{k d}-\left(\mu_{r}^{-1} \sqrt{k^{2}+\lambda_{L}^{-2}}-k\right) e^{-k d}},
\end{aligned}
$$

where $\mathbf{m}(\mathbf{k})=\sum_{i} \mathbf{m}_{i} e^{-i \mathbf{k} \cdot \boldsymbol{\rho}_{i}}$ and where the integrals over $k$ are over the first Brillouin zone of the reciprocal lattice. The expression (28) was obtained in the limit in which one can disregard the lattice structure of the dipole distribution. If such a structure has to be taken into account, then one has to use Ewald summation methods 17 to handle the discrete sums in equation (25).

The expression (28) has two important limits. The first is when $\mu_{r}=1$ and $\lambda_{L} \rightarrow \infty$, or when $d \rightarrow \infty$. We obtain

$$
E=\frac{\mu_{0}}{8 \pi} \int_{B Z} \frac{d^{2} k}{(2 \pi)^{2}} \frac{2 \pi}{k}(\mathbf{k} \cdot \mathbf{m}(\mathbf{k}))(\mathbf{k} \cdot \mathbf{m}(-\mathbf{k}))
$$

which is the familiar result for the dipolar energy of a thin film. The second limit is when the largest contribution to the energy comes from modes $\mathbf{m}(\mathbf{k})$ for which $k \leq L^{-1}$ where $L$ is a length such that $L \gg \lambda_{L} \gg d\left(\right.$ we take $\left.\lambda_{L} \gg d\right)$. In this case, we obtain

$$
E \approx \frac{\mu_{0}}{4 \mu_{r} \lambda_{L}} \int_{k \leq L^{-1}} \frac{d^{2} k}{(2 \pi)^{2}} \frac{(\mathbf{k} \cdot \mathbf{m}(\mathbf{k}))(\mathbf{k} \cdot \mathbf{m}(-\mathbf{k}))}{k^{2}}\left(1+\frac{1}{2}\left(k \lambda_{L}\right)^{2}+O\left(k^{4}\right)\right)
$$


which shows that for $k \ll \lambda_{L}^{-1}$ the energy of the system has the same form as the energy of a system of dipoles in a two dimensional space. Also, comparing equation (28) (with $\mu_{r}=1$ ) with (29), one can conclude that the dipolar interaction has effectively been enhanced with respect to the simple film situation, since the fraction in (28) is always larger than 1 when $\mu_{r}=1$. This result can be easily understood from the fact that the energy given in (28) also includes the kinetic energy of the supercurrent 18 .

The modified dipolar kernel $F(k)=\frac{1}{k} \frac{\left(\sqrt{k^{2}+\lambda_{L}^{-2}}+k\right) e^{k d}+\left(\sqrt{k^{2}+\lambda_{L}^{-2}}-k\right) e^{-k d}}{\left(\sqrt{k^{2}+\lambda_{L}^{-2}}+k\right) e^{k d}-\left(\sqrt{k^{2}+\lambda_{L}^{-2}}-k\right) e^{-k d}}$ given in (28) with $\mu_{r}=1$, is plotted against the kernels $1 / k$ and $1 / k^{2}$, which appear respectively, in (29) and (30), in figure 3 .

The question now arises if one can indeed detect such change of behaviour in the dipolar interaction in artificial superlattices of superconductors and ferromagnetic materials or even in naturally layered systems like $\mathrm{RuSr}_{2} \mathrm{GdCu}_{2} \mathrm{O}_{8-\delta}$ or $\mathrm{RuSr}_{2} \mathrm{Gd}_{1+x} \mathrm{Ce}_{1-x} \mathrm{Cu}_{2} \mathrm{O}_{10}$. The detection of such a change of behaviour relies on the possibility of finding an experimental system which obeys a series of constraints.

Firstly, the London approximation, which was used and which postulates a local relation between the current and the magnetic induction, is only valid sufficiently close to the critical temperature at which the superconducting phase transition occurs. But we believe that the qualitative features of this solution should be valid even when the London approximation is not (i.e. at low temperatures compared to the critical temperature). Physically, one should expect this type of behaviour as long as the superconductor displays the Meissner effect.

Secondly, we have considered a ferromagnetic layer with zero thickness. The solution obtained can only be valid if one can neglect the proximity effect in the ferromagnetic layer, i.e. the induction of superconductivity in the ferromagnetic material by the superconductor 2 . 
Otherwise, in a layer with finite thickness, an effective exchange interaction will be induced between the magnetic moments and this interaction also has to be taken into account. However, this effect is negligible in insulators. Therefore, an appropriate material to choose for the ferromagnetic layer would be a ferromagnetic insulator 49 . We have also neglected the supression of the superconducting order parameter at the boundary between the two media, which always occurs in the presence of a film of finite thickness. In the case of a film made of a ferromagnetic insulator, the de Gennes boundary conditions tell us that the order parameter is effectively quenched to zero at the boundary 50 . Since the London penetration depth depends on this parameter, this will mean that the effective London penetration depth will be greater than the penetration depth measured in the absence of the ferromagnetic film. Nevertheless, the London approximation remains valid (provided that we are working in a weak field situation, see below). The opposite limit, in which the coupling between the superconducting and the ferromagnetic layers is primarily due to the proximity effect has been considered, using a microscopic approach by Radović et al 51 and by Schinz and Schwabl52 using a phenomenological description. In both cases, the problem treated reduces to the one of decoupled thin superconducting layers embedded in a ferromagnet. Experiments done in superlattices of $\mathrm{Fe} / \mathrm{V} 1 \mathrm{O}$ and $\mathrm{Nb} / \mathrm{Gd} 19$ have confirmed these results.

Thirdly, one cannot have any Josephson currents flowing through the insulating junction, otherwise the boundary condition $y_{z}=0$ at $z= \pm d$ is not valid. This implies that the phase difference between the superconducting order parameters of the two superconductors is zero. This is the case for most superconducting systems in equilibrium but in certain junctions containing ferromagnetic materials a non-zero difference between the phases of the superconducting order parameter in each side of the junction, leading to a current in 
equilibrium, has been predicted 53 .

Finally, we have not considered the thermodynamical stability of the system we are working with. This implies that we are working in a weak field situation, i.e. the field produced by the distribution of magnetisation is much lower than the critical field at which the superconducting system undergoes a transition to the intermediate state (in the case of type I superconductors) or to the mixed state (in the case of type II superconductors). One way to achieve this condition is to choose either a magnetic material with a low saturation magnetisation or a superconductor with a high critical field (in the case of type I superconductor), e.g. V, or a high field $H_{c 1}$ (in the case of a type II superconductor), e.g. $\mathrm{Nb}$.

In summary, despite the restrictions pointed above, we think that there is room for believing that one could manufacture systems composed of alternating magnetic and superconducting layers where the above effect could be detected using the methods discussed in the introduction or others.

\section{CONCLUSIONS}

We have computed the magnetic field distribution of an arbitrary distribution of planar magnetisation in a spatial gap between two superconductors. The purpose of this calculation is to provide a simple model for a superlattice of ferromagnetic materials and superconductors where the dipolar interaction between the magnetic moments in the ferromagnetic system is taken into account.

We have also computed the dipolar energy of such a system and we have shown that for low momenta compared to the London inverse penetration depth, the system has a dipolar 
energy which resembles the energy of a magnetisation distribution in 2 dimensions and hence it leads to an enhancement of the dipolar energy compared to that of a simple ferromagnetic film.

As to possible future directions of research one can point out the possibility to consider a treatment of the same problem in the line of what was done in reference 22 , i.e. consider the Landau-Ginzburg theory of the layered system including the free energy of the superconducting system and of the ferromagnetic layer and the interaction between the two via the electromagnetic interaction and the proximity effect, with the appropriate boundary conditions. However, in this case one has to take into account the non-linearity of the problem and the three dimensional character of a solution involving a non-uniform distribution of magnetisation in the plane.

A slightly different model which can also be treated exactly with the methods developed in this paper is the one of a thin ferromagnetic layer between two bulk type II superconductors above $H_{c 1}$, i.e. with flux penetration in the form of an Abrikosov vortex lattice oriented along the $z$ axis. In this case, the boundary conditions (17-19) would be unchanged, but the equation determining $g(\mathbf{r})$, equation (9), would be replaced by an inhomogeneous equation where the role of sources is played by the vortices. Also, the $\pi$ rotational invariance of the system along the $x$ axis is no longer present, but one can still draw useful conclusions from the reflexion properties of the system in the $x y$ plane (seed4 $)$. What makes the model much more difficult to solve is the fact that one cannot exclude the presence of Neumann functions in the Fourier-Bessel integrals which determine $g(\mathbf{r})$ and $\Phi_{M}(\mathbf{r})$, and also that in this case one cannot use the equation (27) as the expression for the total magnetic energy of the system (compare with 4 ). One should nevertheless mention that a method for computing 
the magnetic energy in the presence of vortices has been considered by Erdin et al. case of a thin magnetic film interacting with a thin superconducting film.

Acknowledgements: We acknowledge many helpful discussions with M. Kulić, D. Rainer, H. Braun, H. Kinder, G. Eilenberger, M. A. Santos, C. Bracher, M. Riza and M. Kleber. J.E.S. acknowledges the support of the European Commission, Contract No. ERB-FMBI-CT 97-2816 and from the Deutsche Forschungsgemeinschaft Schwerpunktprogramm 'Strukturgradienten in Kristallen', contract no. Schw 348/12-1 (from 01/03/00). E. F. acknowledges the support of the Deutsche Forschungsgemeinschaft through an Heisenberg fellowship, contract no. FR850/3. E. F. also acknowledges the hospitality of the Institut für Theoretische Physik, LMU München, where part of this work was done. F. S. acknowledges the support of the Deutsche Forschungsgemeinschaft Einzelprojekt Schw. 348/10-1 and of the BMBF Verbundprojekt 03-SC5-TUM0. 


\section{REFERENCES}

${ }^{1}$ V. L. Ginzburg, Soviet Physics JETP 4, 153 (1957).

${ }^{2}$ F. London, Superfluids, (Dover, New York, 1961), Vol. 1.

${ }^{3}$ B. T. Mathias, H. Suhl and E. Corenzwit, Phys. Rev. Lett. 1, 92 (1958).

${ }^{4}$ P. W. Anderson and H. Suhl, Phys. Rev. 116, 898 (1959).

${ }^{5}$ A. A. Abrikosov and L. P. Gor'kov, Sov. Phys. JETP 12, 1243 (1961).

${ }^{6}$ P. G. de Gennes and G. Sarma, J. Appl. Phys. 34, 1380 (1963).

${ }^{7}$ H. Suhl, J. Less-Common Metals 62, 225 (1978).

${ }^{8}$ See L. N. Bulaevskii, A. I. Buzdin, M. L. Kulić and S. V. Panjukov, Adv. Phys. 34, 175 (1985) and references therein.

${ }^{9}$ T. Herrmannsdörfer and F. Pobel, J. Low Temp. Phys. 100, 253 (1995).

10 T. Hermannsdörfer, P. Smeibidl, B. Schröder-Smeibidl and F. Pobel, Phys. Rev. Lett. 74, 1665, (1995).

${ }^{11}$ S. Rehmann, T. Herrmannsdörfer and F. Pobel, Phys. Rev. Lett. 78, 1122 (1997).

12 M. L. Kulić, A. I. Buzdin and L. N. Bulaevskii, Phys. Rev. B 56, 415 (1997).

${ }^{13}$ B. Y. Jin and J. B. Ketterson, Adv. Phys. 38, 189 (1989).

${ }^{14}$ W. Zinn, B. Saftic, N. Rasula, M. Mirabal and J. Köhne, J. Magn. Magn. Mat. 35, 329 (1983).

${ }^{15}$ H. K. Wong and J. B. Ketterson, J. Low Temp. Phys. 63, 139 (1986); H. K. Wong, B. Y. 
Jin, H. Q. Yang, J. B. Ketterson and J. Hilliard, J. Low Temp. Phys. 63, 307 (1986).

${ }^{16}$ P. M. Tedrow, J. E. Tkaczyk and A. Kumar, Phys. Rev. Lett. 56, 1746 (1986).

${ }^{17}$ S. Takahashi and M. Tachiki, Phys. Rev. B 35, 145 (1987).

${ }^{18}$ A. Millis, D. Rainer and J. A. Sauls, Phys. Rev. B 38, 4504 (1988).

${ }^{19}$ C. Strunk, U. Paschen, C. Sürgers and H. v. Löhneysen, Physica B 194-196 2403 (1994);

C. Strunk, C. Sürgers, K. Röhberg and H. v. Löhneysen, Physica B 194-196 2405 (1994).

${ }^{20}$ L. Bauernfeind, W. Widder and H. Braun, Physica C 254, 151 (1995).

${ }^{21}$ C. Bernhard, J. L. Tallon, Ch. Niedermayer, Th. Blasius, A. Golnik, E. Brücher, R. K. Kremer, D. R. Noakes, C. E. Stronach and E. J. Ansaldo, Phys. Rev. B 59, 14099 (1999).

${ }^{22}$ D. J. Pringle, J. L. Tallon, B. G. Walker and H. J. Trodahl, Phys. Rev. B 59, 11679 (1999).

${ }^{23}$ V. G. Hadjiev, A. Fainstein, P. Etchegoin, H. J. Trodahl, C. Bernhard and M. Cardona, Phys. Stat. Sol. (b) 211, R5 (1999).

${ }^{24}$ L. Bauernfeind, W. Widder and H. Braun in Fourth Euro Ceramics (Faenza Editrice S.p.A, Italy, 1995), Vol. 6.

${ }^{25} \mathrm{H}$. Braun, private communication.

${ }^{26}$ See for example, G. Deutscher and P. G. de Gennes in Superconductivity, R. D. Parks, ed. (Dekker, New York, 1969), 1005.

${ }^{27}$ B. D. Josephson, Rev. Mod. Phys. 36, 216 (1964). 
${ }^{28}$ A. Aharony and M. E. Fisher, Phys. Rev. B 8, 3323 (1973); A. Aharony, ibid. 83342 (1973).

${ }^{29}$ A. D. Bruce and A. Aharony, Phys. Rev. B 10, 2078 (1974).

${ }^{30}$ T. Nattermann and S. Trimper, J. Phys. C 9, 2589 (1976).

${ }^{31}$ A. D. Bruce, J. M. Kosterlitz and D. R. Nelson, J. Phys. C 9, 825 (1976); A. D. Bruce, ibid. 10, 419 (1977).

${ }^{32}$ M. A. Santos, J. Phys. C 13, 1205 (1980).

${ }^{33}$ H. S. Kogon and A. D. Bruce, J. Phys. C 15, 5729 (1982).

${ }^{34}$ E. Frey, Dissertation Thesis, Technische Universität München, 1989; E. Frey and F. Schwabl, J. Phys. (Paris) Colloq. 49, C8-1569 (1988); Phys. Rev. B 42, 8261 (1990); ibid. 43, 833 (1991).

${ }^{35}$ R. A. Pelcovits and B. I. Halperin, PRB 19, 4614 (1979).

${ }^{36}$ See for example S. W. Lovesey, Theory of neutron scattering from condensed matter (Clarendon Press, Oxford, 1984).

${ }^{37}$ J. Kötzler, F. Mezei, D. Görlitz, B. Farago, Europhys. Lett. 1, 675 (1986).

${ }^{38}$ P. Böni, D. Görlitz, J. Kötzler and J. L. Martínez, Phys. Rev. B 43, 8755 (1991).

${ }^{39}$ Peter Maier, Diplomarbeit, Technische Universität München, 1998.

${ }^{40}$ Note that in this paper we have considered, for simplicity, a system of planar magnetic moments (an XY model, in the language of field theory). In some ferromagnetic systems this constraint is obeyed but in general one would have to consider a magnetic moment with 
an arbitrary orientation with respect to the $x y$ plane. Due to the linearity of the London equations, such a problem can be splited into the problem which we have solved and the problem of a magnetic moment oriented along the $z$ axis between two superconductors.

${ }^{41}$ A. Kashuba, Phys. Rev. Lett. 73, 2264, (1994).

${ }^{42}$ M. Kardar, G. Parisi and Y. C. Zhang, Phys. Rev. Lett. 56, 889 (1986).

43 J. D. Jackson, Classical Electrodynamics, 3rd ed., (Wiley, New York, 1999).

${ }^{44}$ One can, instead of using the $\pi$ rotation invariance around the $x$ axis, use the reflexion invariance of the problem in the $x y$ plane, which is also valid in the multiple dipole case. But one has to remember that $\mathbf{h}, \mathbf{b}$ and $\mathbf{m}(\boldsymbol{\rho})$ are axial vectors, which makes the analysis more complicated.

${ }^{45}$ M. Schwartz, Principles of Electrodynamics, (Dover, New York, 1987).

${ }^{46}$ It can also be shown from the general expression for the total energy of a system of superconductors treated in the London approximation $⿴ 囗 ⿱ 一 一)$ and of the ferromagnetic layer using equations (1) to (6) and the boundary conditions for $\mathbf{h}$ and $\mathbf{b}$, that the only term which survives is the one given by equation (27).

${ }^{47}$ P. Ewald, Ann. Phys. (Leipzig) 64, 253 (1921).

${ }^{48}$ It can be easily shown that the difference between equation (28) at finite $\lambda_{L}$ and at infinite $\lambda_{L}$ is always positive, which shows that the magnetic energy of the system is always larger in the superconducting state.

${ }^{49}$ D. Rainer, private communication. 
${ }^{50}$ P. G. de Gennes, Rev. Mod. Phys. 36, 225 (1964); O. Entin-Wohlman, Phys. Rev. B 12, 4860 (1975); O. Entin-Wohlman and S. Alexander, J. Low. Temp. Phys. 24, 229 (1976).

${ }^{51}$ Z. Radović, L. Dobrosavljević-Grujić, A. I. Buzdin and J. R. Clem, Phys. Rev. B 38, 2388 (1988).

${ }^{52}$ H. Schinz and F. Schwabl, J. Low Temp. Phys. 88, 347 (1992); J. Mag. Mag. Mat. 93, $303(1991)$.

${ }^{53}$ L. N. Bulaevskii, V. V. Kuzii and A. A. Sobyanin, JETP Lett. 25, 290 (1977).

${ }^{54}$ S. Erdin, A. F. Kayali, I. F. Lyuksyutov and V. L. Pokrosvky, arXiv: cond-mat/0007016 and references therein.

${ }^{55}$ See L. Landau and E. Lifshitz, Electrodynamics of Continuous Media, (Pergamon, Oxford, 1960). 


\section{Figure Captions}

Figure 1. Thin ferromagnetic film between bulk superconductors (schematic).

Figure 2. In-plane distribution of magnetisation between bulk superconductors (schematic).

Figure 3. The modified dipolar kernel $F(k)$ (the continous grey plot) is plotted against the kernels $1 / k$ (the long-dashed plot) and $1 / k^{2}$ (the short-dashed plot). We have taken

$\mu_{r}=1$ and $d=0.1, \lambda_{L}=1$ in $F(k)$ (in arbitrary units). It is seen that the function $F(k)$ interpolates between $1 / k^{2}$ and $1 / k$, the crossover length being the London penetration depth $\lambda_{L}=1$ 


\section{FIGURES}

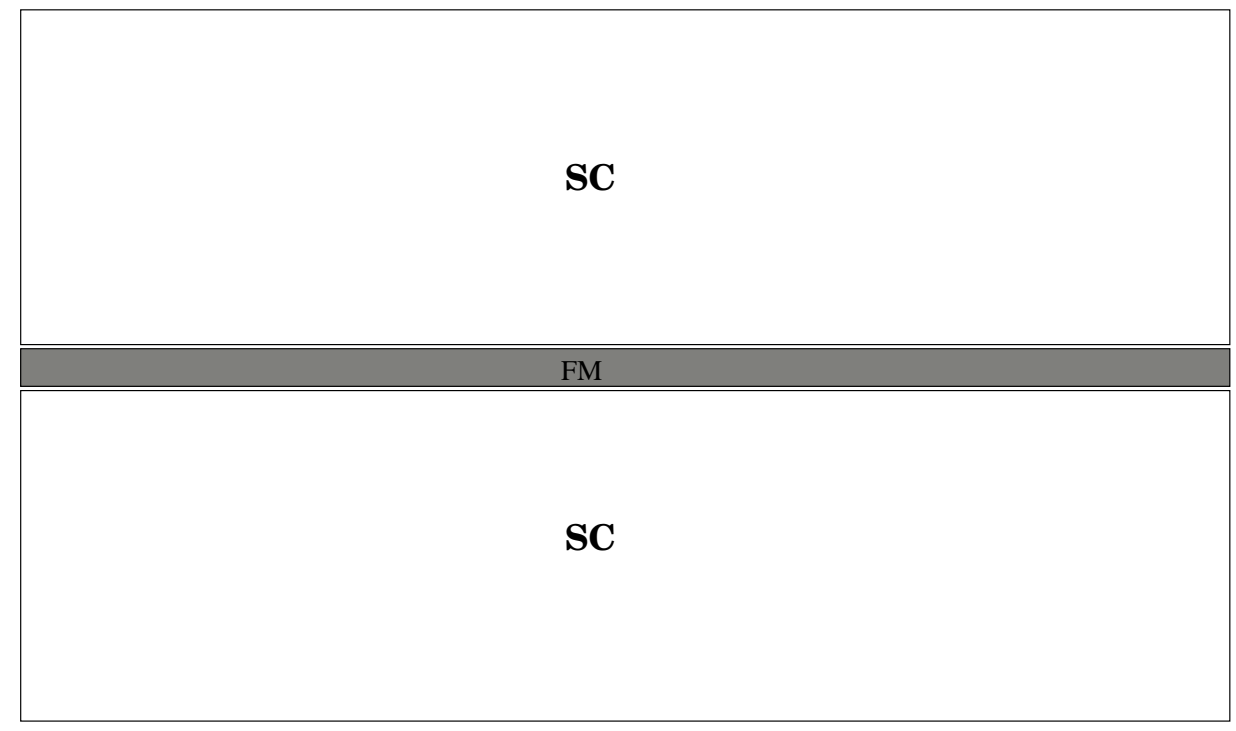

FIG. 1. 


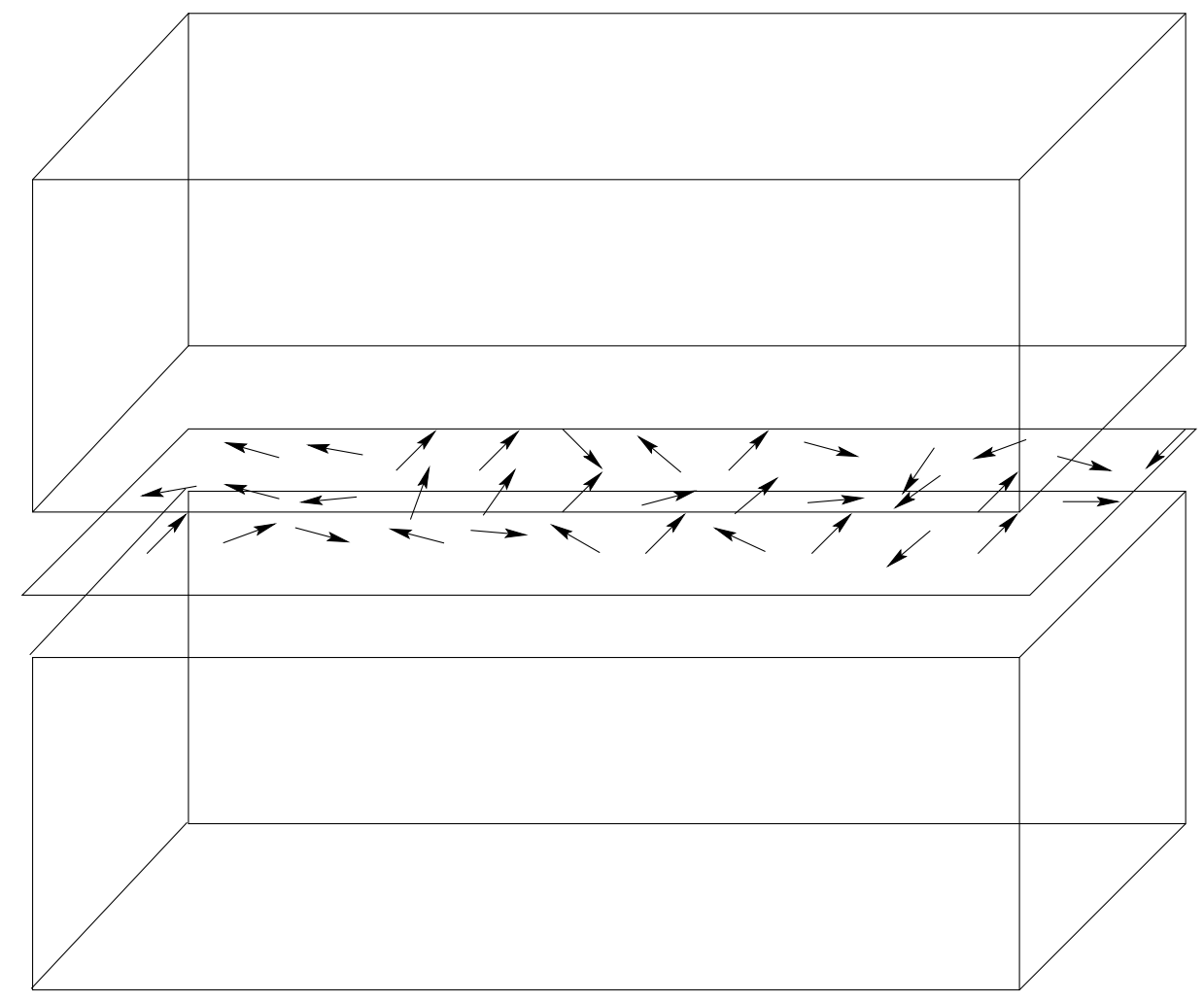

FIG. 2. 


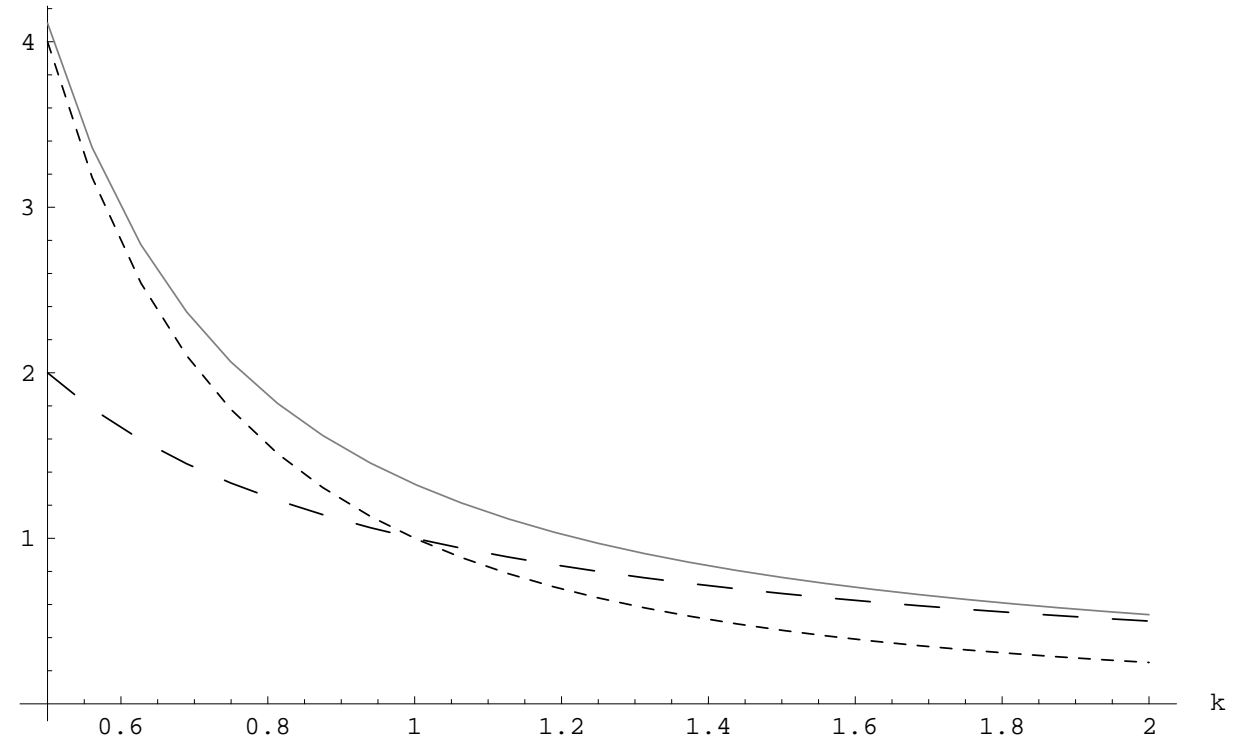

FIG. 3. 